that genuine diffracted $P K P$ waves should nevertheless be observable. The present investigation gives evidence of the existence of these diffracted $P K P$ waves, and at times up to 12 sec. before the $P K I K P$ waves arrive. Thus supplementary proof is given of the fact that the phase $P K I K P$ is unconnected with diffraction, and therefore connected with the presence of an inner core.

The present communication incidentally illustrates the potential value of nuclear explosions to seismological research.

K. E. BULLEN Department of Applied Mathematics, University of Sydney. May 30.

1 Austral. J. Phys., 10, 130 (1957).

2 Bureau Centr. Séism. Internat., A, Fasc. 14, 3 (1936).

${ }_{3}^{3}$ Mon. Not. Roy. Astro. Soc., Geophys. Supp., 4, 552 (1939).

\section{Detection of Inhomogeneities in Trans- parent Plastic Sheet by a Simple Schlieren Technique}

Transparent plastic sheet and film of most types of polymer respond well to the Schlieren technique, and this method can be used to obtain magnified images of inhomogeneities in the material which are normally not detectable by visual inspection. A very simple method based on the Schlieren principle has been developed which requires only an ordinary slide projector and a clean-cut square piece of an opaque material such as cardboard or thin sheet metal the size of which should be somewhat larger than that of the front lens of the projector.

The specimen under investigation is mounted on the slide carrier without cover glasses and projected on to a screen at the desired magnification. The Schlieren effect is produced by partially covering the front lens of the projector with the opaque sheet. Inhomogeneities such as flow lines or particles of different density then become visible on the screen in the form of high-contrast magnified images.

The opaque sheet should be held as close as possible to the front lens. Where the front lens is recessed or hooded, it is advisable to remove the projecting rim. In some cases it will be found advantageous to stop down the light by placing an aperture in front of the condenser lens. The position of the sheet with respect to both the degree of lens coverage and the angular direction of the traversing edge at which maximum contrast is obtained depends on the physical and optical properties of the specimen as well as on the type of projector used, and is best found by trial. For plastic sheet and film, which are usually anisotropic, the optimum position of the edge is at right-angles to the direction of orientation of the specimen.

The effect of this method is shown in the accompanying photographs, which have been taken by placing a photographic plate (Kodak $B 4$ ) in the position of the screen. In Fig. 1, $(a)$ is the image of a cellulose acetate film which was extruded from a non-homogeneous melt, as it appears on the screen on 'straight' projection; (b) shows the appearance of the same area of film with the opaque sheet in position; (c) and (d) are Schlieren pictures of disturbances in similar cellulosic materials due to incorrect manufacturing conditions. The diamond pattern of the flow lines in $(d)$ indicates 'cold flow' due to low production tempera. ture. Fig. 2 is a typical image of polythene film. All reproductions are $\times 20$.

In all cases, the pattern revealed by the Schlieren method was not, or only faintly, recognizable on inspection in transmitted or reflected light. A study of the various patterns can supply useful information about the manufacturing history as well as some rheological properties of the plastic, and has been used for identifying different materials.

Alternatively, the opaque sheet may be replaced by a sheet of 'Polaroid', and another sheet of 'Polaroid', which is 'crossed' with respect to the front sheet, placed between condenser lens and specimen. The effect is the same as that of the single opaque sheet; but this arrangement shows in addition whether any of the inhomogeneities are caused by depolarizing substances such as certain organic fibres, which would appear bright.

Thanks are due to the Directors of British Celanese, Ltd., for permission to publish this communication.

Physics Development Section,

British Celanese, Ltd., Celanese House,

Hanover Square, London, W.1. March 27. 